\title{
Ideal Date Range
}

National Cancer Institute

\section{Source}

National Cancer Institute. Ideal Date Range. NCI Thesaurus. Code C93577.

The date and time span specifying the best time for an activity or event to begin and end. 